\title{
A reliable method for continuously monitoring motor activity in unrestrained squirrel monkeys*
}

\author{
WILLIAM S. LOVE and VINCENT P. HOUSER \\ Psychotropic Drig Laboratory, VA Hospital, Perry Point, Maryland 21902
}

\begin{abstract}
A simple, reliable, and inexpensive method for measuring spontaneous motor activity in unrestrained squirrel monkeys is described. In this method, the home cage is suspended within a wooden enclosure in a way that allows the cage to swing in any lateral direction. This movement is recorded by two brass cylinders connected to the home cage and to the fixed wooden enclosure, respectively. The brass cylinders act as electrical contacts that serve to trigger an electronic recording device. The method is extremely sensitive.
\end{abstract}

The continuous monitoring of motor activity in unrestrained primates presents some methodological problems. Other investigators (Carlton, 1969) have attempted to measure changes in activity level by means of a response lever located in the test chamber, but this method has been justifiably criticized as lacking sufficient sensitivity (Sutterer \& Obrist, 1972). Other methods include the use of photocells or microswitches to sense lateral movement within a specific area. It is difficult to apply either of these two methods to the measurement of activity in unrestrained monkeys in their home cage. The accuracy of the photocell method depends upon the location of the photobeams in reference to the animal. Since individual animals have different patterns of movement, a sufficient degree of sensitivity could be obtained by using many photocells placed on all four walls of the home cage. But use of this technique entails considerable expense. Since the placement of the photocells directly affects the sensitivity of this measure, replication of results from laboratory to laboratory may be a problem.

Similar difficulties are encountered if the home cage is pivoted and microswitches are used at the four corners of the home cage to act as movement sensors. With this method, the placement of the pivot becomes critical 10 insure a balanced system. Since the weights of the individual animal and home cage are variable, replication is difficult both within and between laboratories.

The present report describes a device for measuring spontaneous motor activity in unrestrained squirrel monkeys which operates in a simple and reliable fashion and is inexpensive to construct. More importantly, the data generated should be easily replicable since they are not substantially affected by variability in the movement patterns or weight of the individual animal. This is the case simply because the present system senses any lateral movement irrespective of how these movements are patterned. Furthermore, the home cage is pivoted to swing in response to minimum amounts of lateral force

\footnotetext{
*The authors wish to thank Luther R. Gilliam of the Medical Illustration Service, VA Hospital, Perry Point, Maryland, for perform ing the photographic work.
}

for a distance of 1/64 in. Since almost all movements of squirrel monkeys, irrespective of their weight, are sufficient to produce this movement, variations in animal size do not substantially affect the sensitivity of this device.

Figure 1 is a photograph of the apparatus used to obtain a measure of spontaneous motor activity in unrestrained squirrel monkeys. Although the exact dimensions of this apparatus are not critical to its design, we will provide them to give the reader a point of reference when constructing similar units. The outside wooden enclosure was constructed of $3 / 4$-in. plywood. The outside dimensions of this enclosure were $37 \frac{1}{2}$ in. wide $\times 32$ in. high $\times 30$ in. deep. The home cage consists of a frame constructed from $1 \frac{1 / 2}{2} \times 1 \frac{1}{2} \times 1 / 8$ in. aluminum angle that was covered with galvanized turkey wire $(1 \times 2$ in. mesh $)$.

Figure 1 shows the home cage suspended from the top of the wooden enclosure by four separate aluminum straps connected to the four corners of the cage. These straps were $7^{3 / 4} \mathrm{in}$. long $\times 1$ in. wide $\times 1 / 16$ in. thick. Two holes were drilled at either end of these straps $6 \frac{1}{2} \mathrm{in}$. apart. The tops of the holes were filed to a $90 \mathrm{deg}$ "V" angle so as to form female seats for a knife-edge pivot. The male blade of the pivot was formed by $1 / 4-20$ machine screws filed to form a $30 \mathrm{deg}$ angle. These machine screws passed through the V-shaped holes of the aluminum straps and the holes drilled in the wooden enclosure and home cage. Thus, the machine screws acted to bolt the straps to the wooden enclosure and home cage so that the cage was suspended from the top of the enclosure. The machine screws were not fully tightened at each connection point to enable the cage to swing in any lateral direction. The knife-edge pivots at each connection point provided better centering of the cage in the neutral position than if the pivots were not filed to form angles. As Fig. 1 indicates, a slot was added to the lower 90 deg female pivot on each strap with a large hole in the upper part of the slot; this provided a means of removing the cage for cleaning.

The electrical sensing mechanism was constructed of two concentric brass cylinders. The outer cylinder was 


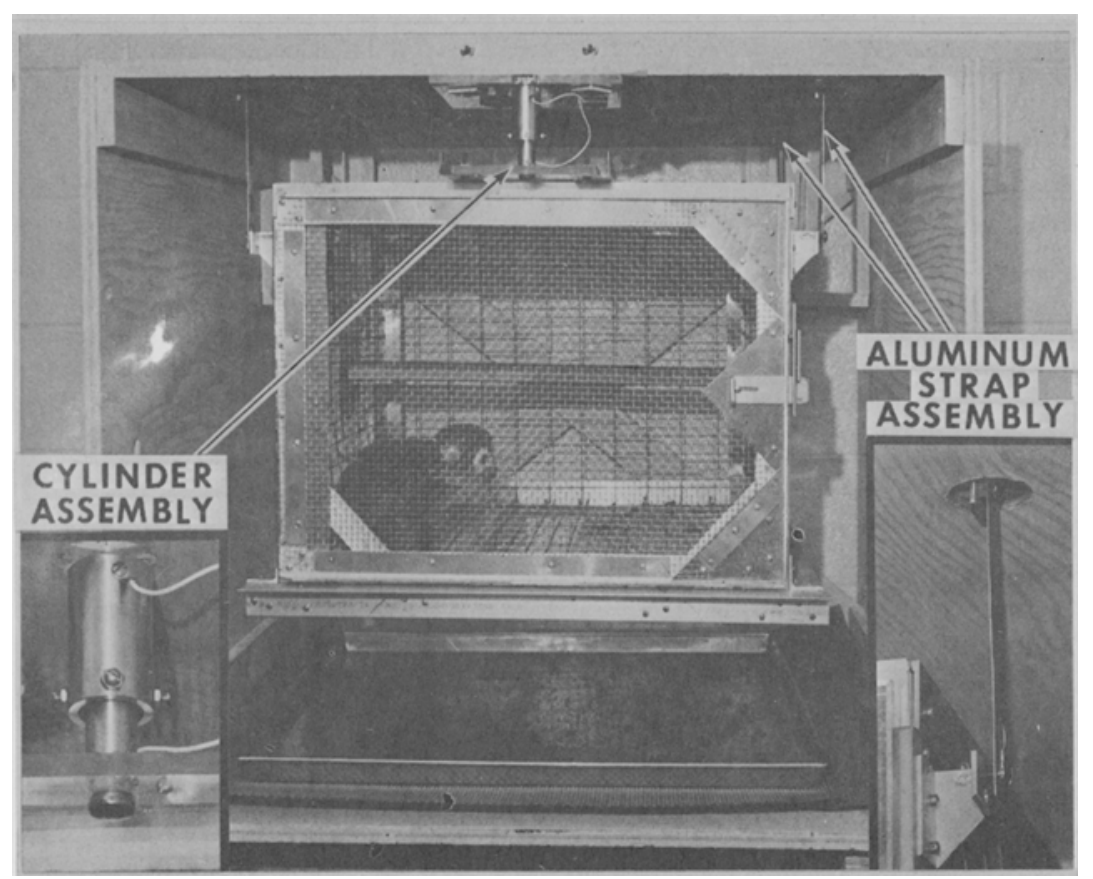

Fig. 1. Photograph of apparatus used to measure spontaneous motor activity in squirrel monkeys in their home cage. The two inserts in the photograph depict the cylinder assembly that detects movement and the aluminum strap assembly, which is used to suspend the restraint chair in a manner that allows it to swing in any lateral direction.

11/4-in. brass rod drilled to a $15 / 16$-in. inside diameter. The inner cylinder was a $3 / 4$-in. brass rod drilled to an inside diameter of $3 / 8$ in. and threaded so that a $3 / 8$-in. bolt could be used to mount the inner cylinder to the plastic plate attached to the cage. The outer cylinder was drilled and tapped to accommodate four 6-32 screws. The screws provided a means of increasing the sensitivity of the apparatus and insured a fine adjustment feature so that the inner cylinder would be equally spaced from the outer cylinder when the cage was in the neutral position. The outer cylinder was attached to a piece of $3 / 8$-in. thick plastic bolted to the top of the wooden enclosure. The plastic had oversized holes through which the bolts passed to connect it to the wooden enclosure. These holes allowed for adjustment of the outer cylinder position so that it remained centered over the inner cylinder when the cage was stationary. The inner cylinder was bolted to another $3 / 8$-in. plastic plate that was bolted to the cage.

This concentric cylinder assembly allows for the detection of any movement within the suspended cage. Animal movements cause the suspended cage to swing slightly. Since the inner ring is connected to the cage, it also moves, bringing it in contact with the machine screws that pass through the stationary outer cylinder. This contact conducts an electrical impulse that can be fed through an electromechanical pulse former and then into a printout counter. Since the machine screws in the outer cylinder can be adjusted to remain 1/64 in. from the inner cylinder, cage movement of $1 / 64$ in. can be detected. A lock nut on the outside portion of the machine screws (Fig. 1) insures that the sensitivity adjustments remain fixed over repeated trials.

Two wooden stops are bolted to the enclosure on either side of the rear of the cage. These stops prevent the rear of the cage from oscillating about the sensing cylinders at the front of the cage, thus averting high spurious activity counts. The stops are placed $3 / 16$ in. from the rear edges of the cage. This prevents the back from swaying more than $3 / 16 \mathrm{in.}$ in either lateral direction.

The reliability of this method has made it possible to collect mean daily activity measures that were reasonably consistent. For example, the mean daily activity counts and standard error of the mean for two squirrel monkeys measured over a 14-day period were as follows: $539.0 \pm 40.3 ; 1,469.0 \pm 76.4$.

\section{REFERENCES}

Carlton, P. Brain acetylocholine and inhibition. In J. T. Tapp (Ed.), Reinforcement and behavior. New York: Academic Press, 1969. Pp. 286-327.

Sutterer, J., \& Obrist, P. A. Heart rate and general activity alterations of dogs during several aversive conditioning procedures. Journal of Comparative \& Physiological Psychology, 1972, 80, 314-326.

(Received for publication December 7, 1973; revision received January 17,1974 .) 